\title{
Nanomaterials for the Local and Targeted Delivery of Osteoarthritis Drugs
}

\author{
Parthiban Chinnagounder Periyasamy, Jeroen C. H. Leijten, Pieter J. Dijkstra, \\ Marcel Karperien, and Janine N. Post
}

Department of Developmental Bioengineering, MIRA Institute for Biomedical Technology and Technical Medicine, Faculty of Science and Technology, University of Twente, 7522 NB Enschede, The Netherlands

Correspondence should be addressed to Janine N. Post, j.n.post@utwente.nl

Received 6 January 2012; Revised 17 February 2012; Accepted 20 February 2012

Academic Editor: Suprakas Sinha Ray

Copyright (C 2012 Parthiban Chinnagounder Periyasamy et al. This is an open access article distributed under the Creative Commons Attribution License, which permits unrestricted use, distribution, and reproduction in any medium, provided the original work is properly cited.

\begin{abstract}
Nanotechnology has found its potential in every possible field of science and engineering. It offers a plethora of options to design tools at the nanometer scale, which can be expected to function more effectively than micro- and macrosystems for specific applications. Although the debate regarding the safety of synthetic nanomaterials for clinical applications endures, it is a promising technology due to its potential to augment current treatments. Various materials such as synthetic polymer, biopolymers, or naturally occurring materials such as proteins and peptides can serve as building blocks for adaptive nanoscale formulations. The choice of materials depends highly on the application. We focus on the use of nanoparticles for the treatment of degenerative cartilage diseases, such as osteoarthritis (OA). Current therapies for OA focus on treating the symptoms rather than modifying the disease. The usefulness of OA disease modifying drugs is hampered by side effects and lack of suitable drug delivery systems that target, deliver, and retain drugs locally. This challenge can be overcome by using nanotechnological formulations. We describe the different nanodrug delivery systems and their potential for cartilage repair. This paper provides the reader basal understanding of nanomaterials and aims at drawing new perspectives on the use of existing nanotechnological formulations for the treatment of osteoarthritis.
\end{abstract}

\section{Introduction}

Osteoarthritis is caused by a multitude of factors, which have not been completely explored and understood. However, the gathered clinical observations and research data on $\mathrm{OA}$ during the last decades help to understand some of the processes involved in the etiology, development, and progression of the disease. The perception that OA is a cartilage-specific disorder is slowly changing towards the view that it is a disorder of the whole joint [2-4]. During OA, apart from articular cartilage, abnormal physiological changes are observed in subchondral bone, synovial membrane, and connective tissue (Figure 1) [5]. Despite the accumulating knowledge of OA, there is currently no US Food and Drug administration approved systemic drug able to modify the disease progression. The clinical practice is, therefore, based on symptomatic treatment aimed at alleviation of pain and surgical replacement of joints with end-stage OA [6]. Drugs that target specific elements of $\mathrm{OA}$ disease progression have been developed, but are associated with severe side effects preventing it from routine clinical use.

Measures of reversing or regenerating the degraded cartilage are being extensively studied, but largely remain in a preclinical phase $[7,8]$. Accumulating knowledge of OA disease mechanisms suggests that abnormal activation of signaling networks plays a leading role in cartilage degradation [9-11] along with other factors such as mechanical loading [12]. In order to rescue cartilage from the catabolic effects of these pathways, the balance of anabolic/catabolic pathways must be restored [11]. Many targets to establish this balance have been previously described [13-15]. Drugs that are specific for these various targets have also been reported [16]. However, the therapeutic success of these disease modifying drugs mainly depends on the delivery route, as most of these drugs are associated with adverse side effects that are not acceptable for nonlife threatening diseases [1]. Local 

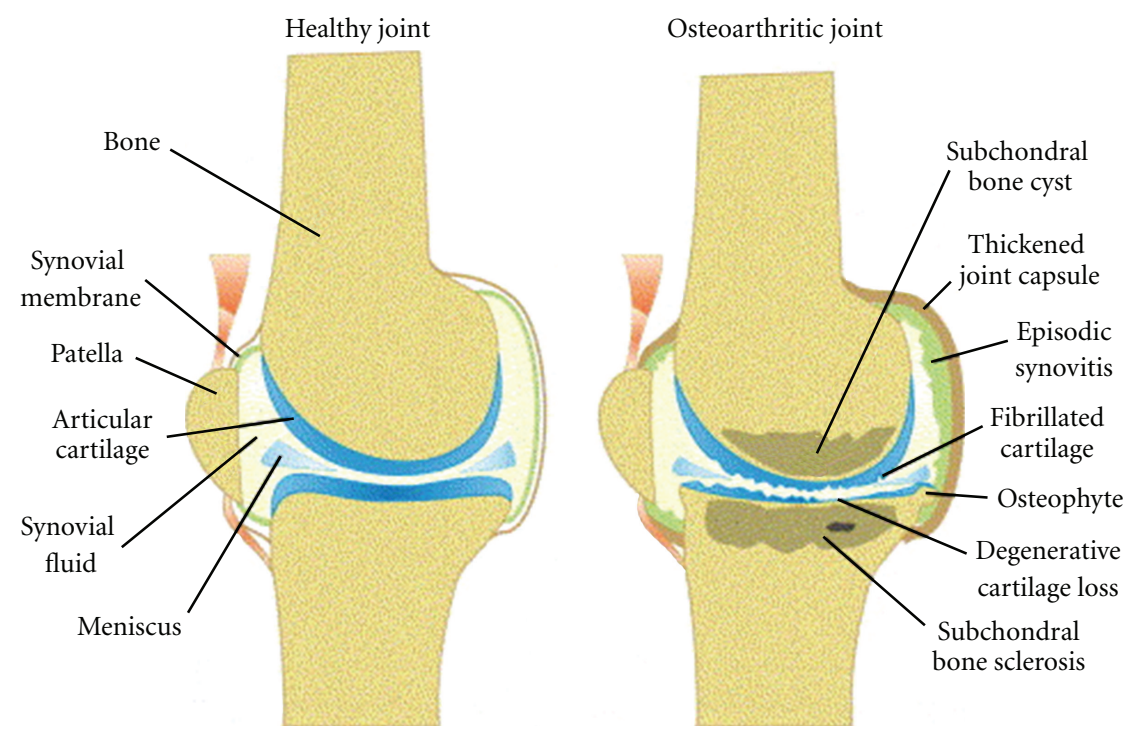

FIgURE 1: Healthy (on left) and Osteoarthritic joints [1]. Joint components such as articular cartilage, subchondral bone, and synovial membrane show abnormal morphology in osteoarthritic joint. Reproduced with permission from Elsevier.

delivery of these drugs using nanoscale carriers might prove essential in circumventing the systemic side effects caused by these drugs. This paper details the potential of nanoparticulate systems for the use in local delivery of osteoarthritis drugs in synovial joints. We describe and discuss the advantages and disadvantages of using micelles (2), liposomes (3), dendrimers (4), and other nanoparticles (5) for intra-articular delivery of drugs in $\mathrm{OA}$, as well as the drugs that can be combined with these vehicles (6).

Nanomaterials are used in the field of medicine in different configurations for a variety of applications, including drug delivery [17], imaging [18], and diagnostics. Cancer treatments have benefited highly from using nanotechnology as many anticancer drugs are toxic by nature, which can cause severe side effects by acting in healthy tissues upon systemic administration [19]. This necessitated the use of nanoscale delivery vehicles in cancer treatment to circumvent these drug side-effects. Nanoscale delivery vehicles have been used successfully to deliver drugs at the site of the tumor. This is facilitated by the enhanced permeability and retention effect of tumors [20]. Passive tissue targeting formulations are an accepted form of nanodrug delivery systems. Various formulations have been created that are focused around the anti-cancer drugs and its targeting ligands. As a result, a large number of nanoparticle systems is available and proven to be effective and are currently in clinical trials. Different nanomaterial configurations include micelles, liposomes, dendrimers, nanoparticles, and macrostructures made of nanoscale materials. Each of these has been used in various applications and can be modified if needed.

Articular cartilage repair strategies can benefit from intra-articular delivery of therapeutic nanoformulations on multiple levels including: improvement in drug retention time, improved bioavailability of drugs, high efficiency at low concentrations, and reduced side effects due to the containment of drugs in the joint space.
To optimize the potential of nanomaterials for osteoarthritis treatments, it is important to realize that the existing nanomaterials require chemical adaptations, which are based on our biological understanding of the synovial joint and its articular cartilage. Therefore, we provide a combinatorial view of both nanomaterials and the biological aspects of the joint.

\section{Micelles}

Micelles are formed when an amphiphilic molecule (having hydrophilic and hydrophobic domains) is dispersed in aqueous medium. The critical micelle concentration is the minimal concentration of the amphiphile in aqueous solution necessary for micelle formation. When a poorly soluble drug is added to this mixture, the hydrophobic domain entraps the drug at its core and protects it from the aqueous medium. The hydrophilic shell stabilizes the formed micelle (Figure 2). For micelles made of amphiphilic co-block polymers, the critical association constant (analogous to critical micelle concentration of surfactant micelles) is very low. This enables continued stability of the micelle even after a large dilution in vivo. The size of the polymeric micelles ranges approximately from $10 \mathrm{~nm}$ to $100 \mathrm{~nm}$ [22]. Depending on the properties of the amphiphile and drug payload, the size of the micelle varies. Use of polymeric micelles as drug delivery vehicles came into practice by preparation of doxorubicin-conjugated block copolymer micelles [23]. The use of polymeric micelles as drug delivery vehicles has become widely accepted and the latest advances in this field have recently been reported [24]. Typically, polymeric micelles are made as injectable formulations. Studies concerning polymeric micelles include, but are not limited to, application in the fields of active targeting anticancer drugs to tumors [25], imaging of various structures in vivo [26], and delivery of nucleic acids to cells [27]. 


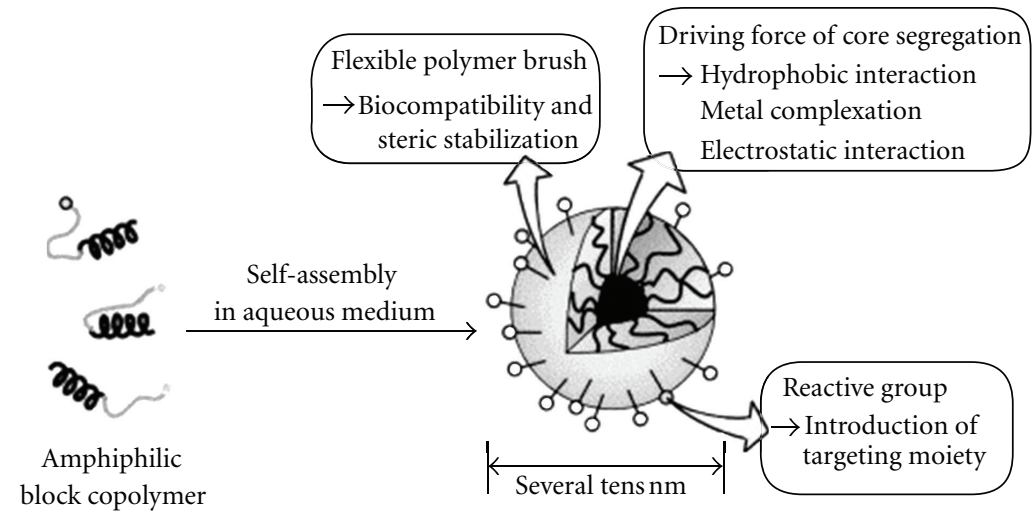

FIGURE 2: Features of polymeric micelles [21]. An amphiphilic copolymer in aqueous medium forms a micelle where the hydrophobic domains come together forming a core and hydrophilic domains forms the shell of the micelle. Hydrophobic drugs are entrapped in the core. Reactive functional groups at the micelle surface can be used to couple ligands for targeted delivery. Reproduced with permission from Elsevier.

TABLe 1: Polymeric micelle formulations in clinical trials [26].

\begin{tabular}{|c|c|c|c|c|}
\hline Clinical phase & Diameter & Block copolymer & Drug & Tissue target \\
\hline \multirow{3}{*}{$\mathrm{I} / \mathrm{II}$} & $20-50 \mathrm{~nm}$ & $\mathrm{DEC} \mathrm{D}(\mathrm{D} \mathrm{J}$ loctide) & Doglitomel & Pancreatic cancer in combination with gemcitabine \\
\hline & 20,501111 & PEG-P(D,L-lactide) & Paclitax & Ovarian cancer in combination with carboplatin \\
\hline & $30 \mathrm{~nm}$ & PEG-Pglu(cisplatin) & Cisplatin & Solid tumors \\
\hline \multirow{4}{*}{ II } & $20 \mathrm{~nm}$ & PEG-PGlu(SN-38) & SN-38 & Breast cancer \\
\hline & $20-50 \mathrm{~nm}$ & $\mathrm{DF} C \mathrm{P}(\mathrm{DU}$ loctidh) & Doclitoyel & Pancreatic cancer \\
\hline & & PEG-P(D,L-lactide) & Pacnitaxel & Non-small-cell lung cancer in combination with carboplatin \\
\hline & $85 \mathrm{~nm}$ & PEG-P(aspartate) & Paclitaxel & Advanced stomach cancer \\
\hline III & $22-27 \mathrm{~nm}$ & Pluronic L61 and F127 & Doxorubicin & $\begin{array}{l}\text { Adenocarcinoma of oesophagus, gastroesophageal junction, } \\
\text { and stomach }\end{array}$ \\
\hline IV & $20-50 \mathrm{~nm}$ & PEG-P(D,L-lactide) & Paclitaxel & Breast cancer \\
\hline
\end{tabular}

Micelles make excellent vehicles because of their structural similarity to adenovirus [28]. For example, the size of animal viruses ranges from 20 to $100 \mathrm{~nm}$, and these viral particles deliver their genome into the cells after escaping the clearance by kidneys and reticuloendothelial system. Viral particles possess the property of cargo protection (viral gene) by having supramolecular assemblies that provide an inner core for cargo and outer shell (Capsid) made of biopolymers that give the stealth-like properties to the particle. This stealth-like property can also be seen as the biocompatibility that is necessary for evading the patient's immune system. Moreover, the transfection efficiency of viral particles is as well attributed to the presence of suitable ligands at the surface for spatial recognition and timely disintegration of the molecular assembly to deliver the payload. Interestingly, micellar transport is analogous to the transport of cholesterol by lipoproteins where the insoluble cholesterol in blood is transported by vehicles of lipoproteins of varying sizes.

From this example, it is evident that micelles mimic phenomena that occur commonly in mammals. Aforementioned structural similarities of micelles with the naturally occurring nanoscale vehicles provide insight into the use of polymeric micelles for application in drug delivery systems. Many strategies to implement the stealth-like properties, targeting properties, and stability have been tried ever since the use of micelles in drug delivery was implicated. Stealth-like property or biocompatibility can be easily achieved by the use of hydrophilic, biocompatible polymers such as polyethylene glycol (PEG), poly(N-vinyl pyrrolidone), poly(N-isopropyl acrylamide) and poly(hydroxypropyl methacrylamide). Among these hydrophilic shell forming polymers PEG is most extensively used. It is nontoxic, hydrophilic, inert in biological fluids and can be coupled to various chemical groups for ligand attachment [29].

Most of the micelle formulations [26] that are currently in various clinical trial phases contain PEG as the hydrophilic segment. These formulations are able to deliver various drugs to distinct tissue targets. They demonstrate reduced systemic toxicity of the drugs and prolonged circulation times compared to drugs without a nanocarrier. Some of the formulations are listed in Table 1 [26]. These formulations do not contain ligand that directs them towards a specific target. Enhanced permeability and retention effects of tumors are exploited in these cases where the micelles accumulate preferentially in tumor vasculature thus increasing the drug concentration in tumors. The robust nature of these formulations in in vivo conditions and encouraging results from their preceding in vitro studies has enabled these micelles to be tested at various phases of clinical trials. This suggests that these formulations, after being replaced with appropriate 
TABLE 2: Targeted micelle formulations.

\begin{tabular}{|c|c|c|c|c|}
\hline Ligand type & Ligand & Target & Copolymer & Reference \\
\hline \multirow{3}{*}{ Antibody } & $\mathrm{mAb} 2 \mathrm{C} 5$ & $\begin{array}{l}\text { Nucleosome-restricted specificity for } \\
\text { different cancer cells }\end{array}$ & PEG- $b$-PE & {$[54]$} \\
\hline & $\mathrm{mAb} C 225$ & EGF receptor & PEG- $b$-PG & {$[55]$} \\
\hline & mAb HD39-SA & $\mathrm{CD} 22$ & $\begin{array}{l}\text { Biotinylated PDMAEMA-b- } \\
\text { DMAEMA-BMA-PAA }\end{array}$ & {$[56]$} \\
\hline \multirow{4}{*}{ Peptide } & $\begin{array}{l}\alpha_{\mathrm{v}} \beta_{3} \text { ligand } \\
(\mathrm{cRGDfK})\end{array}$ & $\alpha_{\mathrm{v}} \beta_{3}$ integrin & PEG- $b-\mathrm{PCL}$ & {$[57]$} \\
\hline & $\mathrm{VPAC}_{2}$ & Vasoactive intestinal peptide receptor & DSPE-PEG $3400-\mathrm{NHS}$ & {$[58]$} \\
\hline & Angiopep-2 & $\begin{array}{l}\text { Lipoprotein receptor-related protein } \\
\text { (LRP) present on the } \mathrm{BBB}\end{array}$ & PE-PEG & {$[59]$} \\
\hline & WYRGRL & Collagen II $\alpha 1$ & Pluronic F-127 & {$[31]$} \\
\hline \multirow{4}{*}{ Others } & \multirow{3}{*}{ Folate } & \multirow{3}{*}{ Folate receptor } & PLGA- $b$-PEG & {$[60]$} \\
\hline & & & mPEG- $b$-PCL & {$[61]$} \\
\hline & & & $\begin{array}{c}\text { PEG }_{3350}-\mathrm{DSPE}: \\
\mathrm{mPEG}_{2300}-\mathrm{DSPE}(1: 100)\end{array}$ & {$[62]$} \\
\hline & Galactosamine & Asialoglycoprotein receptor (ASGP-R) & $\mathrm{PCL}_{67}-\mathrm{PEEP}_{36}$-CDI & {$[63]$} \\
\hline
\end{tabular}

types of drugs, are suitable for use in drug delivery at different anatomical locations such as the synovial joints. Efficiency of the modified formulations has to be validated through appropriate studies. Micelles also have some disadvantages such as concerns over toxicity, storage stability, and limited number of polymers for clinical use [30]. Controlled delivery of hydrophobic drugs greatly benefits micelle formulations, but for incorporation of hydrophilic drugs they appear less suited. For these purposes, modification of the polymeric building blocks might prove to be necessary.

Nonetheless, it is a promising direction to follow in order to utilize the proven methodologies for applications that need better drug administration routes. Table 2 summarizes the ligands of targeted micelle formulations that have been reported. The ligands can be divided into three categories, namely, monoclonal antibodies, peptides, and others. Preferential binding of a ligand to its receptor (overexpressed in a diseased condition or unique to a cell population) is being exploited to improve the targeting efficiency of micelles. Similar approaches can be applied to target a specific component of the synovial joint in order to prevent or block any undesirable mechanism that could potentially initiate, propagate, or exacerbate joint diseases such as osteoarthritis. Some approaches have been described, such as the use of a specific peptide that targets collagen II $\alpha 1$, WYRGRL, [31], and antibodies against cleavage fragments of aggrecanase [32].

\section{Liposomes}

Liposomes are spherical vesicles made of phospholipid bilayers that are comparable to mammalian cell membranes. Liposomes contain an aqueous compartment which can carry molecules that are protected from the external environment. Methods of forming liposomes include dispersing phospholipids in aqueous medium, high pressure extrusion, sonication, detergent dialysis, and so forth [33]. The different types of liposomes include small unilamellar vesicles $(\sim 100 \mathrm{~nm})$ made of a single bilayer, large unilamellar vesicles $(200-800 \mathrm{~nm})$, and multilamellar vesicles $(500-5000 \mathrm{~nm})$ that contain several bilayers in a concentric manner (Figure 3). The liposomes' surface can be modified with polymers to generate long circulating liposomes and or with antibodies to create immunoliposomes that accumulate at sites expressing the targeted antigen [34].

Drug delivery is one of the major fields where liposomes are extensively studied. Consequently, a number of liposomal formulations encapsulating drugs are studied in clinical trials [36]. Liposomes also have been explored for gene delivery [37], where cationic liposomes are made to facilitate the interaction with the cell membranes and nucleic acids [38]. Targeted liposomal formulations using ligands such as folate $[39,40]$, transferrin $[41,42]$, and RGD peptides [43] have previously been reported. MRI and CT imaging applications have also benefited by the use of liposomes carrying contrast agents $[44,45]$. Liposomes can be loaded with both magnetic materials and therapeutic molecules to enable the magneticfield-assisted localization of these particles for imaging and subsequent elicitation of therapeutic effect in the immediate surroundings.

Surface modification using polymers such as PEG prolongs the circulation time of liposomes. This enables the liposomes to escape the clearance by reticuloendothelial system thereby providing better bioavailability. This is particularly useful for injection into the joint cavity since it prevents the opsonization of liposomal formulations by synovial components. Other polymer coatings reported for this purpose include poly-N-vinylpyrrolidones [46, 47], polyvinyl alcohol [48], and branched oligoglycerols [49].

Intra-articular injection of a liposomal formulation containing both dexamethasone and diclofenac has been shown to be effective in reducing inflammation in knee joints of OA rats [50]. This demonstrates that it is possible to intra-articularly inject a carrier that incorporates different drugs 


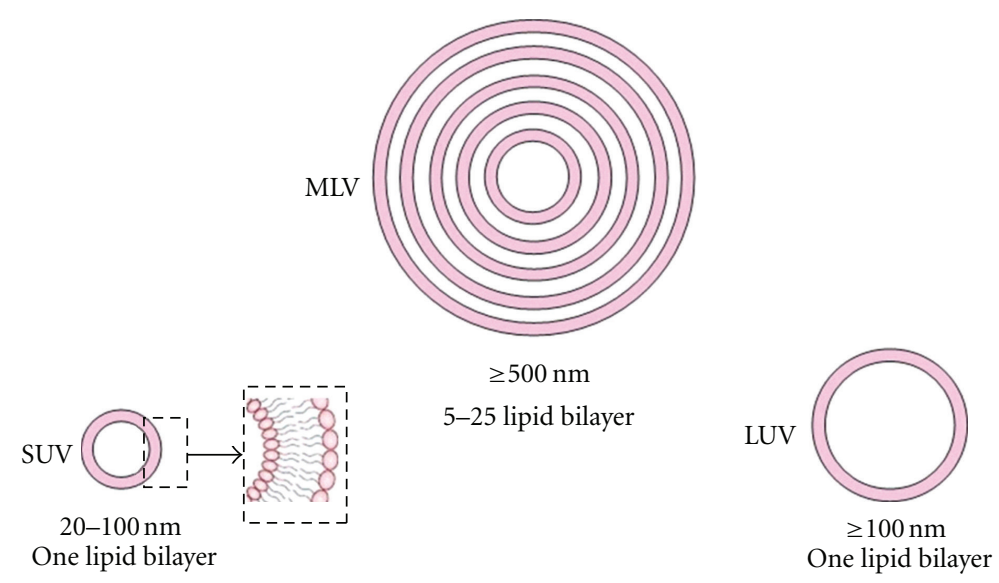

FIGURE 3: Classification of liposomes and their relative sizes. SUV: single unilamellar vesicles, MLV: multilamellar vesicles, LUV: large unilamellar vesicles [35]. Reproduced with permission from Elsevier.

without loss of biological activity. This allows for targeting of different tissues with distinct drug combinations in a straight forward manner.

Advantages of using Liposomes include (1) excellent biocompatibility, (2) easy encapsulation of hydrophilic drugs into their core compartment and hydrophobic drugs into their lipid bilayer, (3) ability to effectively penetrate cell membranes, (4) delivery of drugs into the cell compartments, and (5) versatility in modifying the surface properties in physical and chemical aspects by altering or introducing new components into the lipid bilayer. The systemic use of liposomes has drawbacks such as rapid clearance from the blood, disintegration of the structure, and thereby release of drugs at undesirable site and time. Although local injection within a synovial joint might circumvent these drawbacks, rapid clearance from the synovial fluid remains a challenge for untargeted formulations.

In addition to traditional applications such as drug delivery, liposomes might be used to enhance joint lubrication. Goldberg's group reported that phosphatidylcholine liposomes absorbed to negatively charged mica surfaces reduce the coefficient of friction between surfaces $[51,52]$. In other words, absorption of these liposomes onto two negatively charged surfaces results in less friction upon pressure than the surfaces without these liposomes. This potentially means that when these liposomes are injected into the synovial joints, it can improve lubricity to the joints by binding to the negatively charged cartilage surface. In addition, it has been shown that the lactoferrin loaded positively charged liposomal formulation had higher retention time in the joint when compared to free injected lactoferrin in 24 hours. On the other hand, negatively charged liposomes cleared more rapidly than freely injected drugs [53]. Therefore, it is likely that positively charged liposomes have a higher potential for inflicting an effect in the synovial joint than the formulations with negative or neutral charges. This indicates that specific modifications of nanoparticles can be used to modulate tissue retention times.

\section{Dendrimers}

Dendrimers are a relatively recently developed family of macromolecules that has found its potential in a vast amount of applications. Dendrimers are highly branched synthetic structures with defined components: the core, the shell, and the cavity. The dendrimer size depends on the number of generations, that is, the number of layers it contains (Figure 4). Stepwise increase in size is observed with each generation of a dendrimer. In contrast, the number of functional groups increases exponentially with each generation. The amount of functional groups available in dendrimers is much higher than its linear counterpart, when comparing a linear polymeric chain and a dendrimer of a similar molecular weight. Depending on the dendrimer's generation, the diameter and thus the structure and reactivity varies. As the number of generations increases, steric hindrance of the surface groups allows for only a limited number of chemical groups to be added. This also depends on the property of the chemical group required to be coupled to the dendrimer surface. Nonetheless, dendrimers have a vast amount of reactive groups compared to linear polymers.

Dendrimers are prepared in two different ways. They can be synthesized stepwise from the core diverging to the shell [64] or the branched shell components can be made first and then coupled to a core to form a dendrimer [65]. Click chemistry [66] and thiol-ene chemistry [67] can be used to synthesize different dendrimers. Classic examples of dendrimers are polyamidoamine (PAMAM), poly(L-lysine) (PLL), polypropylenimine (PPI), poly(2,2-bis(hydroxymethyl) propionic acid (bis-MPA), and phosphorous-based dendrimers [68, 69].

The structure and composition of dendrimers can be tightly controlled resulting in the monodispersity of the final product. Monodispersed macromolecules have large numbers of functional groups at their surface, which allows for further defined chemical modifications. In case an antibody is used for such modification, the chemically modified 


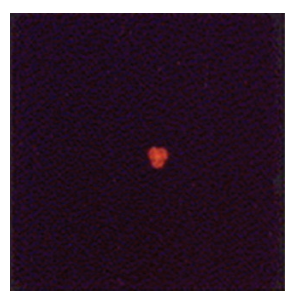

Core

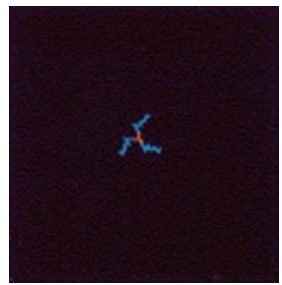

$G=0$

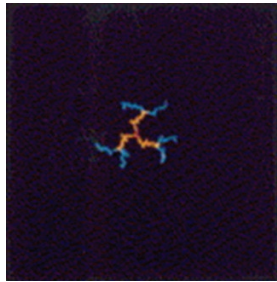

$G=1$

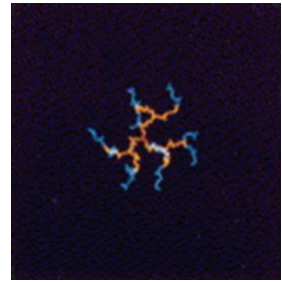

$G=22 \mathrm{~nm}$

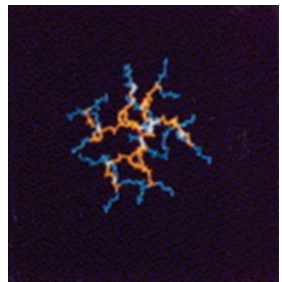

$G=33.1 \mathrm{~nm}$

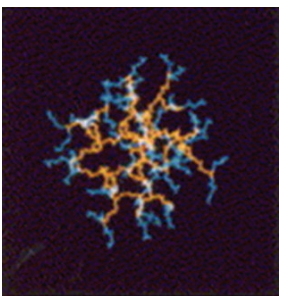

$G=44 \mathrm{~nm}$

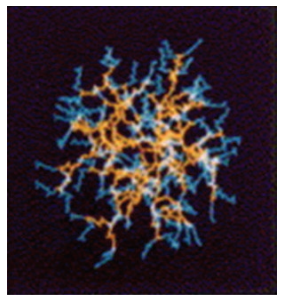

$G=55.3 \mathrm{~nm}$

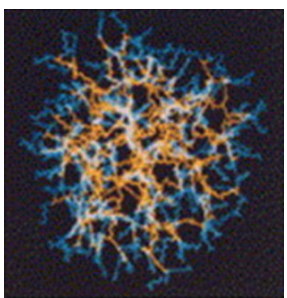

$G=66.7 \mathrm{~nm}$

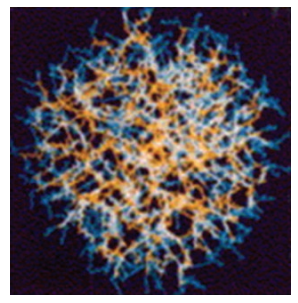

$G=78 \mathrm{~nm}$

FIGURE 4: Graphical presentation of PAMAM dendrimers from core to generation $G=7$ showing the linear increase in diameter and exponential growth of the number of surface groups. In blue, the surface groups are depicted and the core is depicted in yellow/orange [84]. Reproduced with permission from Elsevier.

dendrimer shows much higher affinities towards its corresponding substrate as compared to a macromolecule without ligand [70]. Properties such as high affinity towards substrate and multivalency make dendrimers attractive candidates for targeted delivery vehicles. Moreover, higher generation dendrimers contain internal cavities, which allows for payload to be loaded and protected from the environment until the branches of the dendrimer fall apart.

Although encapsulation of drugs into dendrimers is achieved, the efficiency of drug release remains to be improved. A few studies have reported that the release of more than $70 \%$ encapsulated drugs is in the range of a couple of hours in phosphate buffered saline [71]. Encapsulated drugs in other formulations behave in largely similar manners $[72,73]$. However, the release rate can be highly controlled by the covalent coupling of drugs to the dendrimers and these formulations are called prodrugs. Dendrimers such as PAMAM and PPI have been successfully combined with various anticancer, anti-inflammatory, and antimicrobial drugs either by physical encapsulation or chemical coupling. Detailed information about these drug-dendrimer formulations can be found in the literature [74]. It is essential to know that the type of linkers used to couple the dendrimer and drug has an influence in the rate of the drug release. For example, using PAMAM coupled with paclitaxel using two different linkers such as succinic acid and glutaric acid shows that the former conjugate had a half-life of about 10 hours and the latter did not show a significant drug release in PBS for a week. Nonetheless, a different study showed encouraging results when a glutaric acid linker was used with a different type of dendrimer [75].

Similar to other nanoscale formulations, inclusion of targeting ligands to the surface of dendrimers enhanced the accumulation of targeted dendrimers up to 20 -fold as compared to nontargeted dendrimers [76]. Similar targeted dendrimer formulations have been published $[77,78]$. Dendrimers are proven to be effective for transfection of genes into cells $[79,80]$. Due to the presence of cationic functional groups dendrimers complexed with DNA or RNA fragments enter the cells by receptor-mediated endocytosis and by other routes. The increase in positive charge on the dendrimer surface can cause significant toxicity to the cells and also poor efficiency of transfection. Cationic groups can be neutralized by coupling them with PEG like materials, and this has been proven to be effective in reducing toxicity and improving transfection efficiencies $[80,81]$. Another interesting application of dendrimers that falls under the scope of this paper is their use in MRI imaging of articular cartilage. Nitroxides, a class of contrasting agents that gain positive charge under physiological $\mathrm{pH}$, were linked to PAMAM-dendrimers. These dendrimers are shown to provide information about the articular surface, and potentially also about the distribution of proteoglycans in degenerated cartilage [82]. Absorption of the positively charged dendrimer-Nitroxide by the cartilage tissue enables high contrast in MRI when compared to free nitroxides and gadopentetate dimeglumine. This might prove useful in quantification of regional proteoglycan loss in degenerating joints.

Properties such as cell and ECM penetration make dendrimers attractive candidates for transporting OA drugs to the chondrocytes and cartilage matrix. Recently, the feasibility and potency of dendrimer-based joint treatments has been demonstrated in two established mouse models of autoimmune arthritis [83]. The intravenous injection of phosphorous-based dendrimers decorated with azabisphosphonate $(\mathrm{ABP})$ groups at their surface-reduced expression of proinflammatory cytokines and increased production of anti-inflammatory cytokines. In addition, these dendrimers 
potently inhibited bone resorption and differentiation of monocytes and osteoclasts in vitro. ABP-capped dendrimers inhibited the development of rheumatoid arthritis and reversed paw swelling, clinical scores, and bone erosion in the mice models. Inflammation was completely inhibited showing near normal synovial membranes and intact cartilage after a few weeks. Moreover, dendrimer ABP reduced monocyte differentiation in ex vivo explants of synovial tissue from patients undergoing arthroplastic surgery. This study showed that the ABP-capped dendrimer is capable of reducing proinflammatory factors as well as osteoclast activity both in vivo in mice as well as in vitro in human cells [83], clearly demonstrating the clinical potential of dendrimer-based therapies for joint diseases. Although the potential of dendrimers to target specific joint components is promising, much knowledge remains to be gained before dendrimers can be routinely used in a clinical setting. This is at least partly due to our limited knowledge on in vivo side-effects, time consuming synthesis methods, and the non-bio-degradable nature of many dendrimers.

\section{Other Nanoparticles}

The term nanoparticle is ambiguously used by different groups. All particles at the nanometer scale regardless of their unique chemical, physical, and structural properties could potentially be considered as a nanoparticle. In this paper we will only address the solid nanoparticle formulations made of polymeric materials that do not share physical similarities with other nanomaterials described in this paper. We do not focus on metallic and ceramic nanoparticles as their material properties are likely to further damage the affected joint by erosion.

Polymeric nanoparticles are colloidal materials with sizes ranging from few a nanometers to a few hundred nanometers. The size of the nanoparticles is variable according the type of polymer, bioactive agent, and processing method. Different processing methods include solvent evaporation, interfacial deposition, nanoprecipitation, double emulsion, and spray drying. Biodegradable polymers such as poly-D,Llactide-co-glycolide (PLGA), polylactic acid (PLA), poly- $\varepsilon$ caprolactone (PCL), chitosan, and gelatin are commonly used for nanoparticle preparation. These polymers are highly biocompatible and are used in various drug delivery applications, including cancer and cardiovascular diseases [85, 86]. Drugs are commonly encapsulated in nanoparticles using various methods. For example, the drug can be added to the polymer solution during synthesis or the nanoparticles are synthesized and then incubated in a drug solution to absorb the drug [87]. The drug release profile is highly dependent on the polymer composition. The possible release mechanisms include (1) desorption of the bound drug, (2) release by diffusion, (3) release by polymer erosion, (4) or a combination of the above-mentioned mechanisms. Please note that the drug release profile can be effectively tuned by changing the molecular weight of the polymer [88].

Despite the biocompatibility of the commonly used polymers, it is essential to modify the nanoparticle surface with agents that provide stealth properties that prevent recognition of the patient's immune system. This enables a prolonged retention of drug-loaded nanoparticles in both systemic circulation and intra-articular space. Indeed, PEG surface modified nanoparticles showed a prolonged circulation time compared to nonmodified nanoparticles [89, 90]. Polymers such as PLA and PLGA have been approved for use in humans by the US Food and Drug administration. Therefore, the use of nanoparticles synthesized from these polymers is widely accepted and has previously been reported in several research fields that include gene therapy [91], biomedical imaging [92], drug delivery [93], and cancer therapy [94].

Nanoparticles possess advantages such as biodegradability, controllable size, variety of synthesis methods, and versatility in surface functional groups for coupling of ligands. However, the drug release profile of nanoparticles is typically based on a burst release of the encapsulate drugs. Moreover, nanoparticles are sensitive to opsonization and clearance by reticuloendothelial system.

Owing to the versatile nature of nanoparticles, multiple facets of OA can be addressed by incorporating different drugs into nanoparticles or by incorporating different drugs in different nanoparticles. The potential that nanoparticles hold for the OA treatment is comparable to that of other nanoformulations discussed in this paper.

\section{Potential Formulations for Osteoarthritis Drugs}

Current OA drug therapy recommends the use of nonsteroidal anti-inflammatory drugs (NSAIDs) and cyclooxygenase- 2 inhibitors. NSAIDs are typically anti-inflammatory, analgesic, and antipyretic agents that act by nonspecifically inhibiting the effects of cyclooxygenase 1 (COX-1) and COX2 enzymes, resulting in pain relief. Commonly recommended NSAIDs are Paracetamol, Ibuprofen, and Celecoxib. COX2 mediates inflammation in response to appropriate stimuli [95] and COX-2 expression is observed in degrading joints for which COX-2 inhibitors are widely prescribed. These drugs alleviate pain and improve OA therapy management for a short time span. Use of these drugs is associated with complications such as cardiovascular side effects and gastrointestinal toxicity [96]. Consequently, low doses of these drugs are recommended at the clinical setting. Although topical application of these drugs is possible, they are not potent and cause skin irritation. Besides NSAIDs and COX2 inhibitors, other types of drugs such as cannabinoids, capsaicin analogs, antidepressants, antagonists of kainite receptor, and Bradykinin and antibodies against nerve growth factor are being suggested for OA symptoms and are currently being investigated [97]. Moreover, experimental drugs such as glucosamine sulfate [98], chondroitin sulfate [99], and diacerein [100] have been shown to provide alleviation of symptoms and to have disease modifying effects. Despite the introduction of new compounds, the systemic delivery of drugs is likely also to cause side effects, which are not acceptable for treatment of a nonlife threatening disease with relatively mild complaints particularly in an early stage of 
TABLE 3: Proposed assignment of different nanocarriers for delivery of active drugs to different targets within a synovial joint.

\begin{tabular}{|c|c|c|}
\hline Target & Desired properties of nanocarrier & Potentially suitable nanocarrier \\
\hline Cartilage matrix & $\begin{array}{l}\text { (i) Size smaller than } 38 \mathrm{~nm}^{*} \\
\text { (ii) Positive surface charge } \\
\text { (iii) Ability to couple peptide ligands (WYRGRL) to target collagen II } \alpha 1\end{array}$ & Micelles, Dendrimers \\
\hline Subchondral bone & $\begin{array}{l}\text { (i) Size smaller than } 38 \mathrm{~nm}^{*} \\
\text { (ii) Positive surface charge } \\
\text { (iii) Ability to couple peptide ligands (WYRGRL) to target collagen II } \alpha 1 \text { to } \\
\text { make ECM as a drug reservoir for drugs that can reach subchondral bone } \\
\text { in case of early OA } \\
\text { (iv) Ability to target hydroxyapatite of subchondral bone in case of advance } \\
\text { OA where subchondral bone is exposed due to cartilage degradation }\end{array}$ & Micelles, Dendrimers \\
\hline Cartilage surface & $\begin{array}{l}\text { (i) Variable sizes can be used but if penetration into cartilage has to be } \\
\text { avoided, sizes greater than } 60 \mathrm{~nm} \text { are recommended } \\
\text { (ii) Positive surface charge } \\
\text { (iii) Ability to couple antibodies against epitopes of cartilage degradations } \\
\text { such as VDIPEN and NITEGE }\end{array}$ & $\begin{array}{l}\text { Liposomes, Dendrimers of higher } \\
\text { generation, Micelles, Nanoparticles }\end{array}$ \\
\hline Synovial membrane & $\begin{array}{l}\text { (i) Variable size can be used but if penetration into cartilage has to be } \\
\text { avoided, size greater than } 60 \mathrm{~nm} \text { is recommended } \\
\text { (ii) Ability to retain in intra-articular space with a targeting aspect towards } \\
\text { a synovial joint component and subsequent uptake by synovial fibroblast }\end{array}$ & $\begin{array}{l}\text { Liposomes, Dendrimers of higher } \\
\text { generation, Micelles, Nanoparticles }\end{array}$ \\
\hline Intra articular space & $\begin{array}{l}\text { (i) Variable size can be used but if penetration into cartilage has to be } \\
\text { avoided, sizes greater than } 60 \mathrm{~nm} \text { are recommended } \\
\text { (ii) Ability to form complexes with synovial fluid components that can help } \\
\text { in retention of nanocarrier in intra-articular space }\end{array}$ & $\begin{array}{l}\text { Liposomes, Dendrimers of higher } \\
\text { generation, Micelles, Nanoparticles }\end{array}$ \\
\hline Infrapatellar fat pad & (i) Lipophilic properties that allow preferential absorption by fat tissue & $\begin{array}{l}\text { Liposomes, Dendrimers of higher } \\
\text { generation, Micelles, Nanoparticles }\end{array}$ \\
\hline
\end{tabular}

the disease. It is important to realize that the joints are amenable for intra-articular injections. Therefore, it is intuitive that restriction of such drugs to synovial joint eliminates these side effects. The lack of an adequate delivery system prevents potentially beneficial disease modifying drugs from routine clinical use. For example, inhibiting the activity of p38 MAPK, a signal transduction kinase molecule, can prevent cartilage degeneration and provide pain relief [101, 102]. Several p38 MAPK inhibiting drugs are identified and have demonstrated disease modifying effects in various clinical trials. However, these drugs cause cardiac, neuronal, and hepatic complications, which prevent the routine systemic use of these drugs [103]. These side effects would be omitted when these drugs are used with an adaptive drug delivery system that delivers and retains the active drugs within the tissues of the synovial joint. Moreover, such approaches can be used to target multiple signaling pathways in the joint.

Most of the nanoformulations in clinical trials are not target specific. With this in mind, it is to be noted that particles smaller than $5 \mu \mathrm{m}$ are rapidly cleared, as the synovial joint lacks a basement membrane [104]. The therapeutic efficiency of NSAIDs nanoformulations can be improved by enhancing its containment in the synovial joint. One of the ways to improve the retention of these formulations is by the use of positively charged carriers, which prolong the retention time within the synovial joint [53]. Therefore, it can be anticipated that drug efficacy can be improved with minimal side effects and lower drug dosages upon adapting the existing technologies. Consequently, the amounts of the drug able to leak into the systemic circulation will be too low to elicit side effects in other tissues. Moreover, as lower amounts of drugs are required to obtain a similar effect it may improve cost-effectiveness of osteoarthritis drugs.

Within the synovial joint, there are different targets that can be (in)activated to regain control over the catabolic shift. Importantly, the ability to reach the precise anatomical location of these targets will largely determine the therapeutic success. For example, the cellular targets could be on the cell surface, cytosol, nucleus, or intercellular space. These targets include inflammatory cytokines [10], signal transduction pathways [16], gene expression [105], and proteolytic enzymes [106]. The main challenge in intra-articular drug delivery is to prolong the retention time of drugs within the synovial joint. The versatile nature of the different nanoscale carriers makes them highly suitable for targeting and retaining drugs at different loci of the synovial joint (Table 3). For specific osteoarthritis drugs, it may prove unnecessary to directly target cells as the extracellular matrix can potentially be utilized as a drug reservoir. Drugs can be released in the vicinity of the cell and still be available for cellular uptake. This approach is able to deliver a higher amount of drug over a prolonged period of time compared to cell-targeted approaches. In 2008, Rothenburg et al. suggested using a cartilage targeted system in which a nanoparticle was coupled to a small peptide fragment that binds collagen IIa1, which resulted in effective targeting of articular cartilage [31]. 
Moreover, antibodies that bind epitopes of cartilage degradation such as VDIPEN and NITEGE could be used to target degrading cartilage [32]. OA is associated with changes in the subchondral bone, and it is currently a challenge to effectively deliver drugs to this tissue. Using the extracellular matrix as a drug reservoir might overcome this hurdle. It is important to realize that the mesh size of collagen II fibrillar networks is approximately $60 \mathrm{~nm}$ [107], and the spacing between side chains of the proteoglycan network is approximately $20 \mathrm{~nm}$ [108]. Consequently, only nanoparticles of sufficiently small size allow for the penetration into the cartilaginous matrix. Indeed, Rothenfluh et al. showed that nanoparticles of $38 \mathrm{~nm}$ penetrated the cartilage matrix, while nanoparticles of $98 \mathrm{~nm}$ could not [31]. During the early stages of OA, collagen II can be targeted for both cartilage and subchondral bone. However, in advanced stages the subchondral bone is exposed to the intra-articular space due to cartilage erosion. In such cases, hydroxyapatite can be targeted for the nanoparticles to reach subchondral bone. Furthermore, a degree of positive charge could potentially enable the nanocarrier to interact with cartilage matrix. The negative charge provided by sulfated proteoglycans would allow for the electrostatic interaction between the nanocarrier and the matrix.

Anabolic triggers are typically products of articular cartilage, but the catabolic triggers that degrade the articular cartilage are derived from multiple tissues of the joint. The tissues include articular cartilage, subchondral bone, synovium, meniscus, and the infrapatellar fat pad [109]. As a consequence, it remains questionable whether targeting a single symptom or tissue would eventually prove sufficient in treatment of OA. It may be argued that as multiple components of the joint are involved in OA pathology, each with different mechanisms of action, a strategy that aims at different mechanisms and tissues might prove inevitable for therapeutic success in treating OA. Nanomaterials are able to provide a highly controllable platform that allows local delivery of drug combinations to target each of these tissues by injecting a mixture of specifically adapted nanoparticles.

To target distinct tissues, different nanomaterials can be functionalized with appropriate ligands. Each nanomaterial can contain a combination of drugs, peptide, gene fragments, or antibodies to perform a specific function within the targeted tissue. To design such a multifactorial drug delivery system, the following factors should be taken into account: (1) amounts and types of drugs required, (2) type of carrier suitable for the drugs, (3) the carriers ability to reach the target with respect to size, charge, and so forth, (4) type of ligand required for targeting, (5) release profile of the drug, and (6) fate of the carrier after drug release.

The synovial membrane and infrapatellar fat pad contribute to OA by secreting factors that are able to degrade cartilage [10]. Therefore, they can be considered as interesting therapeutic targets. For example, Inflammation of the synovial membrane is associated with OA [110]. Existing evidence suggests that inflammatory cytokines, for example, IL-4 and IL-13, proteolytic enzymes-for example, MMP's and ADAMTS - pain mediators, and nitrous oxide are being released from the inflamed synovial membrane. This ultimately leads to cartilage degradation. Moreover, chronic synovitis is associated with enhanced vascularity of the tissue that propagates the infiltration of macrophages. This perpetuates the inflammation in the synovial membrane. Therefore, it might be possible to prevent or slow down OA progression by blocking the catabolic trigger from the synovial membrane. Any untargeted nanocarrier formulation could potentially be used to deliver drugs to the synovial membrane. Due to the increased vascularity and recruitment of macrophages, the internalization of nanocarriers is enhanced. Nontargeted particles will be cleared by the lymphatic drainage. Chronic synovitis impairs the lymphatic drainage, which is likely to help retain the nanocarriers within the joint space.

Finally, it is important to realize that nanomaterials contain possible therapeutic potentials that are nondrug based. For example, loss of lubrication between the cartilage surfaces could potentially lead to stress-induced arthritis. Treatment using the positively liposomal formulation by Goldberg et al. [52] can be used to append joint lubrication. In some cases, the synovial fluid could also be used as a reservoir of nanoparticle formulations.

\section{Conclusion}

The notion that nanomaterials can be used for drug delivery is well established. However, its utilization remains to be fully integrated in many areas of investigation including the field of osteoarthritis research. Local delivery by nanomaterials can potentially limit or even prevent current side-effects of OA drugs. Osteoarthritis is a multitissue disease, and nanomaterials are an ideal platform to design combinatorial drug therapies that stimulate or inhibit specific pathways in targeted tissues. Straightforward adaptations to the existing technologies can provide the necessary modifications to optimize osteoarthritis drug delivery and improve the therapeutic outcomes.

\section{Authors' Contribution}

P. Chinnagounder Periyasamy and J. C. H. Leijten contributed equally.

\section{Acknowledgments}

The authors gratefully acknowledge the support of the TeRM Smart Mix Program of the Netherlands Ministry of Economic Affairs and the Netherlands Ministry of Education, Culture and Science. Part of the research is funded by project P2.02 OAcontrol of the research program of the Biomedical Materials Institute, cofunded by the Dutch Ministry of Economic Affairs.

\section{References}

[1] N. Gerwin, C. Hops, and A. Lucke, "Intraarticular drug delivery in osteoarthritis," Advanced Drug Delivery Reviews, vol. 58, no. 2, pp. 226-242, 2006. 
[2] M. Attur, J. Samuels, S. Krasnokutsky, and S. B. Abramson, "Targeting the synovial tissue for treating osteoarthritis (OA): where is the evidence?" Best Practice and Research, vol. 24, no. 1, pp. 71-79, 2010.

[3] R. J. Lories and F. P. Luyten, "The bone-cartilage unit in osteoarthritis," Nature Reviews Rheumatology, vol. 7, no. 1, pp. 43-49, 2011.

[4] S. Suri and D. A. Walsh, "Osteochondral alterations in osteoarthritis," Bone. In press.

[5] M. B. Goldring and S. R. Goldring, "Osteoarthritis," Journal of Cellular Physiology, vol. 213, no. 3, pp. 626-634, 2007.

[6] J. W. J. Bijlsma, F. Berenbaum, and F. P. J. G. Lafeber, "Osteoarthritis: an update with relevance for clinical practice," The Lancet, vol. 377, no. 9783, pp. 2115-2126, 2011.

[7] L. Kock, C. C. van Donkelaar, and K. Ito, "Tissue engineering of functional articular cartilage: the current status," Cell and Tissue Research, vol. 347, no. 3, pp. 613-627, 2012.

[8] A. H. Reddi, J. Becerra, and J. A. Andrades, "Nanomaterials and hydrogel scaffolds for articular cartilage regeneration," Tissue Engineering-Part B, vol. 17, no. 5, pp. 301-305, 2011.

[9] J. Bertrand, C. Cromme, D. Umlauf, S. Frank, and T. Pap, "Molecular mechanisms of cartilage remodelling in osteoarthritis," International Journal of Biochemistry and Cell Biology, vol. 42, no. 10, pp. 1594-1601, 2010.

[10] M. Kapoor, J. Martel-Pelletier, D. Lajeunesse, J. P. Pelletier, and H. Fahmi, "Role of proinflammatory cytokines in the pathophysiology of osteoarthritis," Nature Reviews Rheumatology, vol. 7, no. 1, pp. 33-42, 2011.

[11] M. B. Mueller and R. S. Tuan, "Anabolic/Catabolic balance in pathogenesis of osteoarthritis: identifying molecular targets," $P M \& R$, vol. 3, no. 6, supplement 1, pp. S3-S11, 2011.

[12] T. P. Andriacchi and A. Mündermann, "The role of ambulatory mechanics in the initiation and progression of knee osteoarthritis," Current Opinion in Rheumatology, vol. 18, no. 5, pp. 514-518, 2006.

[13] M. J. Alcaraz, J. Megías, I. García-Arnandis, V. Clérigues, and M. I. Guillén, "New molecular targets for the treatment of osteoarthritis," Biochemical Pharmacology, vol. 80, no. 1, pp. 13-21, 2010.

[14] C. Beyer and G. Schett, "Novel targets in bone and cartilage," Best Practice and Research, vol. 24, no. 4, pp. 489-496, 2010.

[15] K. B. Marcu, M. Otero, E. Olivotto, R. M. Borzi, and M. B. Goldring, "NF- $\kappa \mathrm{B}$ signaling: multiple angles to target OA," Current Drug Targets, vol. 11, no. 5, pp. 599-613, 2010.

[16] L. A. J. O’Neill, “Targeting signal transduction as a strategy to treat inflammatory diseases," Nature Reviews Drug Discovery, vol. 5, no. 7, pp. 549-563, 2006.

[17] M. Talekar, J. Kendall, W. Denny, and S. Garg, "Targeting of nanoparticles in cancer: drug delivery and diagnostics," AntiCancer Drugs, vol. 22, no. 10, pp. 949-962, 2011.

[18] X. Chi, D. Huang, Z. Zhao, Z. Zhou, Z. Yin, and J. Gao, "Nanoprobes for in vitro diagnostics of cancer and infectious diseases," Biomaterials, vol. 33, no. 1, pp. 189-206, 2012.

[19] C. Shanholtz, "Acute life-threatening toxicity of cancer treatment," Critical Care Clinics, vol. 17, no. 3, pp. 483-502, 2001.

[20] T. Lammers, F. Kiessling, W. E. Hennink, and G. Storm, "Drug targeting to tumors: principles, pitfalls and (pre-) clinical progress," Journal of Controlled Release. In press.

[21] K. Kataoka, A. Harada, and Y. Nagasaki, "Block copolymer micelles for drug delivery: design, characterization and biological significance," Advanced Drug Delivery Reviews, vol. 47, no. 1, pp. 113-131, 2001.
[22] M. F. Francis, M. Cristea, and F. M. Winnik, "Polymeric micelles for oral drug delivery: why and how," Pure and Applied Chemistry, vol. 76, no. 7-8, pp. 1321-1335, 2004.

[23] M. Yokoyama, G. S. Kwon, T. Okano, Y. Sakurai, T. Seto, and K. Kataoka, "Preparation of micelle-forming polymer-drug conjugates," Bioconjugate Chemistry, vol. 3, no. 4, pp. 295301, 1992.

[24] U. Kedar, P. Phutane, S. Shidhaye, and V. Kadam, "Advances in polymeric micelles for drug delivery and tumor targeting," Nanomedicine, vol. 6, no. 6, pp. 714-729, 2010.

[25] A. Mahmud, X. B. Xiong, H. M. Aliabadi, and A. Lavasanifar, "Polymeric micelles for drug targeting," Journal of Drug Targeting, vol. 15, no. 9, pp. 553-584, 2007.

[26] C. Oerlemans, W. Bult, M. Bos, G. Storm, J. F. W. Nijsen, and W. E. Hennink, "Polymeric micelles in anticancer therapy: targeting, imaging and triggered release," Pharmaceutical Research, pp. 1-21, 2010.

[27] K. Osada, R. J. Christie, and K. Kataoka, "Polymeric micelles from poly(ethylene glycol)-poly(amino acid) block copolymer for drug and gene delivery," Journal of the Royal Society Interface, vol. 6, no. 3, pp. S325-S339, 2009.

[28] K. Kataoka, G. S. Kwon, M. Yokoyama, T. Okano, and Y. Sakurai, "Block copolymer micelles as vehicles for drug delivery," Journal of Controlled Release, vol. 24, no. 1-3, pp. 119132, 1993.

[29] K. Miyata, R. J. Christie, and K. Kataoka, "Polymeric micelles for nano-scale drug delivery," Reactive and Functional Polymers, vol. 71, no. 3, pp. 227-234, 2011.

[30] H. Chen, C. Khemtong, X. Yang, X. Chang, and J. Gao, "Nanonization strategies for poorly water-soluble drugs," Drug Discovery Today, vol. 16, no. 7-8, pp. 354-360, 2011.

[31] D. A. Rothenfluh, H. Bermudez, C. P. O'Neil, and J. A. Hubbell, "Biofunctional polymer nanoparticles for intraarticular targeting and retention in cartilage," Nature Materials, vol. 7, no. 3, pp. 248-254, 2008.

[32] I. I. Singer, D. W. Kawka, E. K. Bayne et al., "VDIPEN, a metalloproteinase-generated neoepitope, is induced and immunolocalized in articular cartilage during inflammatory arthritis," The Journal of Clinical Investigation, vol. 95, no. 5, pp. 2178-2186, 1995.

[33] R. A. Schwendener, "Liposomes in biology and medicine," Advances in Experimental Medicine and Biology, vol. 620, pp. 117-128, 2007.

[34] V. P. Torchilin, "Recent advances with liposomes as pharmaceutical carriers," Nature Reviews Drug Discovery, vol. 4, no. 2, pp. 145-160, 2005.

[35] F. Yang, C. Jin, Y. Jiang et al., "Liposome based delivery systems in pancreatic cancer treatment: from bench to bedside," Cancer Treatment Reviews, vol. 37, no. 8, pp. 633642, 2011.

[36] M. Slingerland, H.-J. Guchelaar, and H. Gelderblom, "Liposomal drug formulations in cancer therapy: 15 years along the road," Drug Discovery Today, vol. 17, no. 3-4, pp. 160166, 2012.

[37] M. P. Czech, M. Aouadi, and G. J. Tesz, "RNAi-based therapeutic strategies for metabolic disease," Nature Reviews Endocrinology, vol. 7, no. 8, pp. 473-484, 2011.

[38] R. B. Campbell, B. Ying, G. M. Kuesters, and R. Hemphill, "Fighting cancer: from the bench to bedside using second generation cationic liposomal therapeutics," Journal of Pharmaceutical Sciences, vol. 98, no. 2, pp. 411-429, 2009.

[39] A. Gabizon, H. Shmeeda, A. T. Horowitz, and S. Zalipsky, "Tumor cell targeting of liposome-entrapped drugs with phospholipid-anchored folic acid-PEG conjugates," 
Advanced Drug Delivery Reviews, vol. 56, no. 8, pp. 11771192, 2004.

[40] Y. Lu and P. S. Low, "Folate-mediated delivery of macromolecular anticancer therapeutic agents," Advanced Drug Delivery Reviews, vol. 54, no. 5, pp. 675-693, 2002.

[41] H. Li and Z. M. Qian, "Transferrin/transferrin receptormediated drug delivery," Medicinal Research Reviews, vol. 22, no. 3, pp. 225-250, 2002.

[42] G. Zhai, J. Wu, B. Yu, C. Guo, X. Yang, and R. J. Lee, "A transferrin receptor-targeted liposomal formulation for docetaxel," Journal of Nanoscience and Nanotechnology, vol. 10, no. 8, pp. 5129-5136, 2010.

[43] R. Srinivasan, R. E. Marchant, and A. S. Gupta, "In vitro and in vivo platelet targeting by cyclic RGD-modified liposomes," Journal of Biomedical Materials Research-Part A, vol. 93, no. 3, pp. 1004-1015, 2010.

[44] H. Fattahi, S. Laurent, F. Liu, N. Arsalani, L. V. Elst, and R. N. Muller, "Magnetoliposomes as multimodal contrast agents for molecular imaging and cancer nanotheragnostics," Nanomedicine, vol. 6, no. 3, pp. 529-544, 2011.

[45] W. J. M. Mulder, G. J. Strijkers, G. A. F. van Tilborg, A. W. Griffioen, and K. Nicolay, "Lipid-based nanoparticles for contrast-enhanced MRI and molecular imaging," NMR in Biomedicine, vol. 19, no. 1, pp. 142-164, 2006.

[46] V. P. Torchilin, T. S. Levchenko, K. R. Whiteman et al., "Amphiphilic poly-N-vinylpyrrolidones: synthesis, properties and liposome surface modification," Biomaterials, vol. 22, no. 22, pp. 3035-3044, 2001.

[47] I. A. Yamskov, A. N. Kuskov, K. K. Babievsky et al., "Novel liposomal forms of antifungal antibiotics modified by amphiphilic polymers," Applied Biochemistry and Microbiology, vol. 44, no. 6, pp. 624-628, 2008.

[48] K. Nakano, Y. Tozuka, and H. Takeuchi, "Effect of surface properties of liposomes coated with a modified polyvinyl alcohol (PVA-R) on the interaction with macrophage cells," International Journal of Pharmaceutics, vol. 354, no. 1-2, pp. 174-179, 2008.

[49] A. Ishihara, M. Yamauchi, H. Kusano et al., "Preparation and properties of branched oligoglycerol modifiers for stabilization of liposomes," International Journal of Pharmaceutics, vol. 391, no. 1-2, pp. 237-243, 2010.

[50] I. Elron-Gross, Y. Glucksam, and R. Margalit, "Liposomal dexamethasone-diclofenac combinations for local osteoarthritis treatment," International Journal of Pharmaceutics, vol. 376, no. 1-2, pp. 84-91, 2009.

[51] R. Goldberg, A. Schroeder, Y. Barenholz, and J. Klein, "Interactions between adsorbed hydrogenated soy phosphatidylcholine (HSPC) vesicles at physiologically high pressures and salt concentrations," Biophysical Journal, vol. 100, no. 10, pp. 2403-2411, 2011.

[52] R. Goldberg, A. Schroeder, G. Silbert, K. Turjeman, Y. Barenholz, and J. Klein, "Boundary lubricants with exceptionally low friction coefficients based on 2D close-packed phosphatidylcholine liposomes," Advanced Materials, vol. 23, no. 31, pp. 3517-3521, 2011.

[53] M. Trie, C. Guillen, D. M. Vaughan et al., "Liposomes as possible carriers for lactoferrin in the local treatment of inflammatory diseases," Experimental Biology and Medicine, vol. 226, no. 6, pp. 559-564, 2001.

[54] V. P. Torchilin, A. N. Lukyanov, Z. Gao, and B. Papahadjopoulos-Sternberg, "Immunomicelles: targeted pharmaceutical carriers for poorly soluble drugs," Proceedings of the National Academy of Sciences of the United States of America, vol. 100, no. 10, pp. 6039-6044, 2003.
[55] J. Vega, S. Ke, Z. Fan, S. Wallace, C. Charsangavej, and C. Li, "Targeting doxorubicin to epidermal growth factor receptors by site-specific conjugation of C225 to poly(L-glutamic acid) through a polyethylene glycol spacer," Pharmaceutical Research, vol. 20, no. 5, pp. 826-832, 2003.

[56] M. C. Palanca-Wessels, A. J. Convertine, R. Cutler-Strom et al., "Anti-CD22 antibody targeting of $\mathrm{pH}$-responsive micelles enhances small interfering RNA delivery and gene silencing in lymphoma cells," Molecular Therapy, vol. 19, no. 8, pp. 1529-1537, 2011.

[57] N. Nasongkla, X. Shuai, H. Ai et al., "cRGD-functionalized polymer micelles for targeted doxorubicin delivery," Angewandte Chemie-International Edition, vol. 43, no. 46, pp. 6323-6327, 2004.

[58] O. M. Y. Koo, I. Rubinstein, and H. Önyüksel, "Actively targeted low-dose camptothecin as a safe, long-acting, disease-modifying nanomedicine for rheumatoid arthritis," Pharmaceutical Research, vol. 28, no. 4, pp. 776-787, 2011.

[59] K. Shao, R. Huang, J. Li et al., "Angiopep-2 modified PE-PEG based polymeric micelles for amphotericin B delivery targeted to the brain," Journal of Controlled Release, vol. 147, no. 1, pp. 118-126, 2010.

[60] H. S. Yoo and T. G. Park, "Folate receptor targeted biodegradable polymeric doxorubicin micelles," Journal of Controlled Release, vol. 96, no. 2, pp. 273-283, 2004.

[61] E. K. Park, S. B. Lee, and Y. M. Lee, "Preparation and characterization of methoxy poly(ethylene glycol)/poly( $\varepsilon$-caprolactone) amphiphilic block copolymeric nanospheres for tumor-specific folate-mediated targeting of anticancer drugs," Biomaterials, vol. 26, no. 9, pp. 1053-1061, 2005.

[62] X. Han, J. Liu, M. Liu et al., "9-NC-loaded folate-conjugated polymer micelles as tumor targeted drug delivery system: preparation and evaluation in vitro," International Journal of Pharmaceutics, vol. 372, no. 1-2, pp. 125-131, 2009.

[63] Y. C. Wang, X. Q. Liu, T. M. Sun, M. H. Xiong, and J. Wang, "Functionalized micelles from block copolymer of polyphosphoester and poly(eopen-caprolactone) for receptor-mediated drug delivery," Journal of Controlled Release, vol. 128, no. 1, pp. 32-40, 2008.

[64] G. R. Newkome and C. D. Shreiner, "Poly(amidoamine), polypropylenimine, and related dendrimers and dendrons possessing different $1 \rightarrow 2$ branching motifs: an overview of the divergent procedures," Polymer, vol. 49, no. 1, pp. 1-173, 2008.

[65] C. J. Hawker and J. M. J. Fréchet, "Preparation of polymers with controlled molecular architecture. A new convergent approach to dendritic macromolecules," Journal of the American Chemical Society, vol. 112, no. 21, pp. 7638-7647, 1990.

[66] M. J. Joralemon, R. K. O’Reilly, J. B. Matson, A. K. Nugent, C. J. Hawker, and K. L. Wooley, "Dendrimers clicked together divergently," Macromolecules, vol. 38, no. 13, pp. 5436-5443, 2005.

[67] K. L. Killops, L. M. Campos, and C. J. Hawker, "Robust, efficient, and orthogonal synthesis of dendrimers via thiolene "click" chemistry," Journal of the American Chemical Society, vol. 130, no. 15, pp. 5062-5064, 2008.

[68] L. Brauge, G. Magro, A. M. Caminade, and J. P. Majoral, "First divergent strategy using two AB2 unprotected monomers for the rapid synthesis of dendrimers," Journal of the American Chemical Society, vol. 123, no. 27, pp. 6698-6699, 2001.

[69] M. Slany, A. M. Caminade, and J. P. Majoral, "Specific functionalization on the surface of dendrimers," Tetrahedron Letters, vol. 37, no. 50, pp. 9053-9056, 1996. 
[70] P. Wu, M. Malkoch, J. N. Hunt et al., "Multivalent, bifunctional dendrimers prepared by click chemistry," Chemical Communications, no. 46, pp. 5775-5777, 2005.

[71] A. K. Patri, J. F. Kukowska-Latallo, and J. R. Baker, “Targeted drug delivery with dendrimers: comparison of the release kinetics of covalently conjugated drug and non-covalent drug inclusion complex," Advanced Drug Delivery Reviews, vol. 57, no. 15, pp. 2203-2214, 2005.

[72] M. Liu, K. Kono, and J. M. J. Fréchet, "Water-soluble dendritic unimolecular micelles: their potential as drug delivery agents," Journal of Controlled Release, vol. 65, no. 1-2, pp. 121-131, 2000.

[73] C. Kojima, K. Kono, K. Maruyama, and T. Takagishi, "Synthesis of polyamidoamine dendrimers having poly(ethylene glycol) grafts and their ability to encapsulate anticancer drugs," Bioconjugate Chemistry, vol. 11, no. 6, pp. 910-917, 2000.

[74] S. Svenson, "Dendrimers as versatile platform in drug delivery applications," European Journal of Pharmaceutics and Biopharmaceutics, vol. 71, no. 3, pp. 445-462, 2009.

[75] J. Lim, A. Chouai, S. T. Lo, W. Liu, X. Sun, and E. E. Simanek, "Design, synthesis, characterization, and biological evaluation of triazine dendrimers bearing paclitaxel using ester and ester/disulfide linkages," Bioconjugate Chemistry, vol. 20, no. 11, pp. 2154-2161, 2009.

[76] A. Quintana, E. Raczka, L. Piehler et al., "Design and function of a dendrimer-based therapeutic nanodevice targeted to tumor cells through the folate receptor," Pharmaceutical Research, vol. 19, no. 9, pp. 1310-1316, 2002.

[77] T. Dutta, H. B. Agashe, M. Garg, P. Balasubramanium, M. Kabra, and N. K. Jain, "Poly (propyleneimine) dendrimer based nanocontainers for targeting of efavirenz to human monocytes/macrophages in vitro," Journal of Drug Targeting, vol. 15, no. 1, pp. 89-98, 2007.

[78] W. Yang, Y. Cheng, T. Xu, X. Wang, and L. P. Wen, "Targeting cancer cells with biotin-dendrimer conjugates," European Journal of Medicinal Chemistry, vol. 44, no. 2, pp. 862-868, 2009.

[79] B. H. Zinselmeyer, S. P. Mackay, A. G. Schatzlein, and I. F. Uchegbu, "The lower-generation polypropylenimine dendrimers are effective gene-transfer agents," Pharmaceutical Research, vol. 19, no. 7, pp. 960-967, 2002.

[80] F. Tack, A. Bakker, S. Maes et al., "Modified poly(propylene imine) dendrimers as effective transfection agents for catalytic DNA enzymes (DNAzymes)," Journal of Drug Targeting, vol. 14, no. 2, pp. 69-86, 2006.

[81] D. Luo, K. Haverstick, N. Belcheva, E. Han, and W. M. Saltzman, "Poly(ethylene glycol)-conjugated PAMAM dendrimer for biocompatible, high-efficiency DNA delivery," Macromolecules, vol. 35, no. 9, pp. 3456-3462, 2002.

[82] C. S. Winalski, S. Shortkroff, E. Schneider, H. Yoshioka, R. V. Mulkern, and G. M. Rosen, "Targeted dendrimer-based contrast agents for articular cartilage assessment by MR imaging," Osteoarthritis and Cartilage, vol. 16, no. 7, pp. 815-822, 2008.

[83] M. Hayder, M. Poupot, M. Baron et al., "A phosphorus-based dendrimer targets inflammation and osteoclastogenesis in experimental arthritis," Science Translational Medicine, vol. 3, no. 81, Article ID 81ra35, 2011.

[84] S. Svenson and D. A. Tomalia, "Dendrimers in biomedical applications-reflections on the field," Advanced Drug Delivery Reviews, vol. 57, no. 15, pp. 2106-2129, 2005.

[85] S. Acharya and S. K. Sahoo, "PLGA nanoparticles containing various anticancer agents and tumour delivery by EPR effect," Advanced Drug Delivery Reviews, vol. 63, no. 3, pp. 170-183, 2011.

[86] J. M. Chan, L. Zhang, R. Tong et al., "Spatiotemporal controlled delivery of nanoparticles to injured vasculature," Proceedings of the National Academy of Sciences of the United States of America, vol. 107, no. 5, pp. 2213-2218, 2010.

[87] K. S. Soppimath, T. M. Aminabhavi, A. R. Kulkarni, and W. E. Rudzinski, "Biodegradable polymeric nanoparticles as drug delivery devices," Journal of Controlled Release, vol. 70, no. 12, pp. 1-20, 2001.

[88] M. F. Zambaux, F. Bonneaux, R. Gref, E. Dellacherie, and C. Vigneron, "Preparation and characterization of protein Cloaded PLA nanoparticles," Journal of Controlled Release, vol. 60, no. 2-3, pp. 179-188, 1999.

[89] D. E. Owens and N. A. Peppas, "Opsonization, biodistribution, and pharmacokinetics of polymeric nanoparticles," International Journal of Pharmaceutics, vol. 307, no. 1, pp. 93102, 2006.

[90] S. Y. Kim and Y. M. Lee, "Taxol-loaded block copolymer nanospheres composed of methoxy poly(ethylene glycol) and poly( $\varepsilon$-caprolactone) as novel anticancer drug carriers," Biomaterials, vol. 22, no. 13, pp. 1697-1704, 2001.

[91] S. Prabha and V. Labhasetwar, "Nanoparticle-mediated wildtype p53 gene delivery results in sustained antiproliferative activity in breast cancer cells," Molecular Pharmaceutics, vol. 1, no. 3, pp. 211-219, 2004.

[92] S. K. Nune, P. Gunda, P. K. Thallapally, Y. Y. Lin, M. L. Forrest, and C. J. Berkland, "Nanoparticles for biomedical imaging," Expert Opinion on Drug Delivery, vol. 6, no. 11, pp. 1175-1194, 2009.

[93] A. Mahapatro and D. K. Singh, "Biodegradable nanoparticles are excellent vehicle for site directed in-vivo delivery of drugs and vaccines," Journal of Nanobiotechnology, vol. 9, article 55, 2011.

[94] Y. Liu, H. Miyoshi, and M. Nakamura, "Nanomedicine for drug delivery and imaging: a promising avenue for cancer therapy and diagnosis using targeted functional nanoparticles," International Journal of Cancer, vol. 120, no. 12, pp. 2527-2537, 2007.

[95] D. A. Willoughby, A. R. Moore, and P. R. Colville-Nash, "COX-1, COX-2, and COX-3 and the future treatment of chronic inflammatory disease," The Lancet, vol. 355, no. 9204, pp. 646-648, 2000.

[96] E. M. Antman, J. S. Bennett, A. Daugherty, C. Furberg, H. Roberts, and K. A. Taubert, "Use of nonsteroidal antiinflammatory drugs: an update for clinicians: a scientific statement from the American Heart Association," Circulation, vol. 115, no. 12, pp. 1634-1642, 2007.

[97] A. Dray and S. J. Read, "Arthritis and pain. Future targets to control osteoarthritis pain," Arthritis Research and Therapy, vol. 9, no. 3, article 212, 2007.

[98] G. Herrero-Beaumont, J. A. Román Ivorra, M. D. C. Trabado et al., "Glucosamine sulfate in the treatment of knee osteoarthritis symptoms: a randomized, double-blind, placebo-controlled study using acetaminophen as a side comparator," Arthritis and Rheumatism, vol. 56, no. 2, pp. 555-567, 2007.

[99] L. M. Wildi, J.-P. Raynauld, J. Martel-Pelletier et al., "Chondroitin sulphate reduces both cartilage volume loss and bone marrow lesions in knee osteoarthritis patients starting as early as 6 months after initiation of therapy: a randomised, double-blind, placebo-controlled pilot study using MRI," Annals of the Rheumatic Diseases, vol. 70, no. 6, pp. 982-989, 2011. 
[100] K. Pavelka, T. Trč, K. Karpaš et al., "The efficacy and safety of diacerein in the treatment of painful osteoarthritis of the knee: a randomized, multicenter, double-blind, placebo-controlled study with primary end points at two months after the end of a three-month treatment period," Arthritis and Rheumatism, vol. 56, no. 12, pp. 4055-4064, 2007.

[101] C. Böhm, S. Hayer, A. Kilian et al., "The $\alpha$-isoform of p38 MAPK specifically regulates arthritic bone loss," Journal of Immunology, vol. 183, no. 9, pp. 5938-5947, 2009.

[102] K. K. Brown, S. A. Heitmeyer, E. B. Hookfin et al., "P38 MAP kinase inhibitors as potential therapeutics for the treatment of joint degeneration and pain associated with osteoarthritis," Journal of Inflammation, vol. 5, article 22, 2008.

[103] M. R. Lee and C. Dominguez, "MAP kinase p38 inhibitors: clinical results and an intimate look at their interactions with p38 $\alpha$ protein," Current Medicinal Chemistry, vol. 12, no. 25, pp. 2979-2994, 2005.

[104] S. H. R. Edwards, "Intra-articular drug delivery: the challenge to extend drug residence time within the joint," Veterinary Journal, vol. 190, no. 1, pp. 15-21, 2011.

[105] T. Nakasa, Y. Nagata, K. Yamasaki, and M. Ochi, "A mini-review: microRNA in arthritis," Physiological Genomics, vol. 43, no. 10, pp. 566-570, 2011.

[106] A. L. Clutterbuck, K. E. Asplin, P. Harris, D. Allaway, and A. Mobasheri, "Targeting matrix metalloproteinases in inflammatory conditions," Current Drug Targets, vol. 10, no. 12, pp. 1245-1254, 2009.

[107] W. D. Comper, Cartilage: Molecular Aspects, CRC Press, 1991.

[108] P. A. Torzilli, J. M. Arduino, J. D. Gregory, and M. Bansal, "Effect of proteoglycan removal on solute mobility in articular cartilage," Journal of Biomechanics, vol. 30, no. 9, pp. 895-902, 1997.

[109] T. Ushiyama, T. Chano, K. Inoue, and Y. Matsusue, "Cytokine production in the infrapatellar fat pad: another source of cytokines in knee synovial fluids," Annals of the Rheumatic Diseases, vol. 62, no. 2, pp. 108-112, 2003.

[110] J. Sellam and F. Berenbaum, "The role of synovitis in pathophysiology and clinical symptoms of osteoarthritis," Nature Reviews Rheumatology, vol. 6, no. 11, pp. 625-635, 2010. 

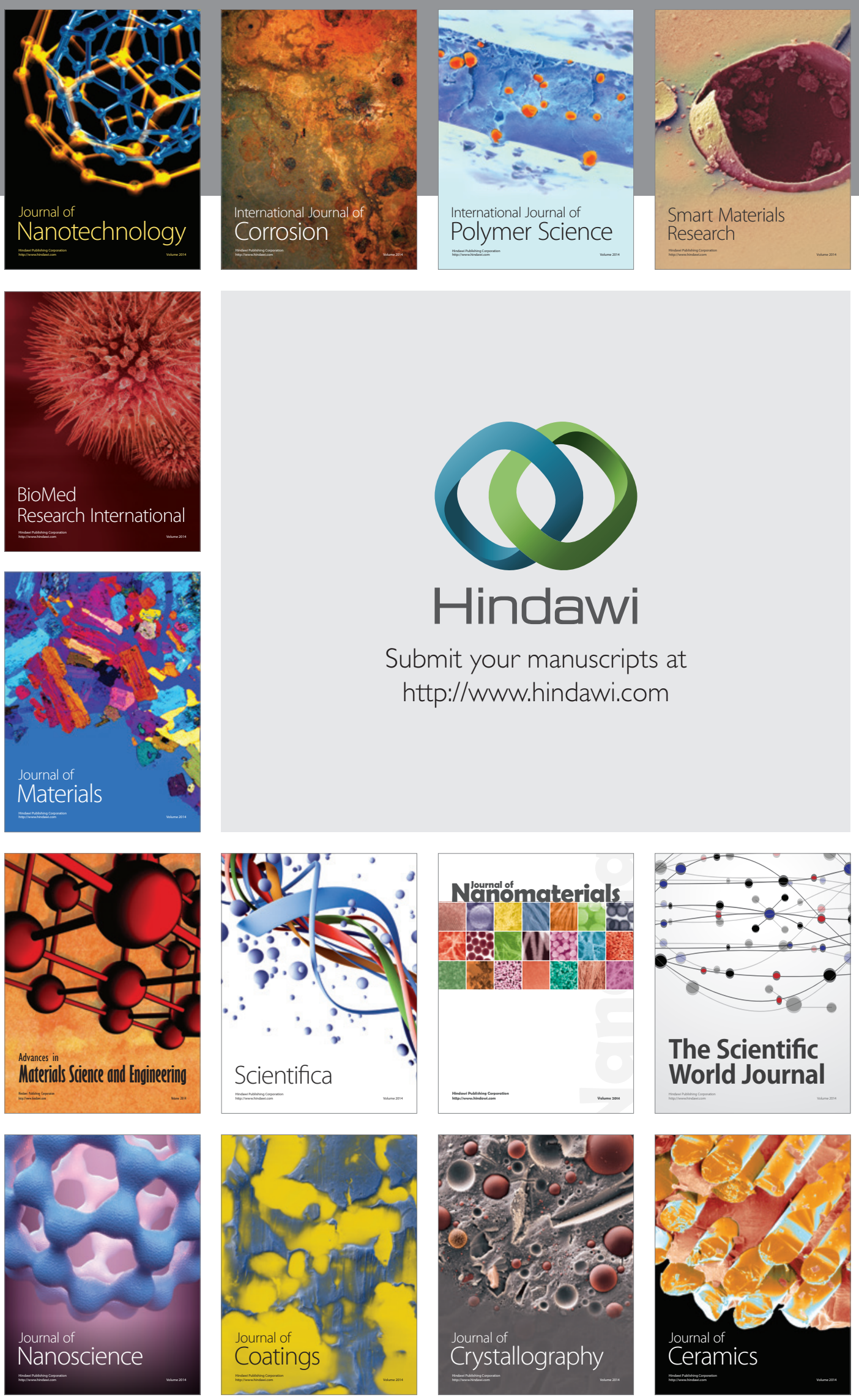

The Scientific World Journal

Submit your manuscripts at

http://www.hindawi.com

\section{World Journal}

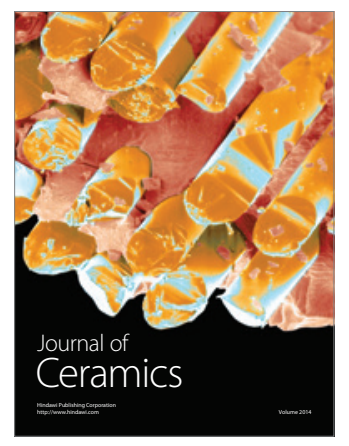

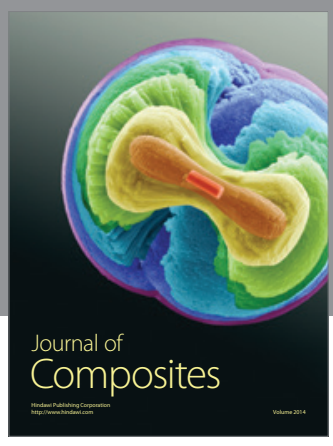
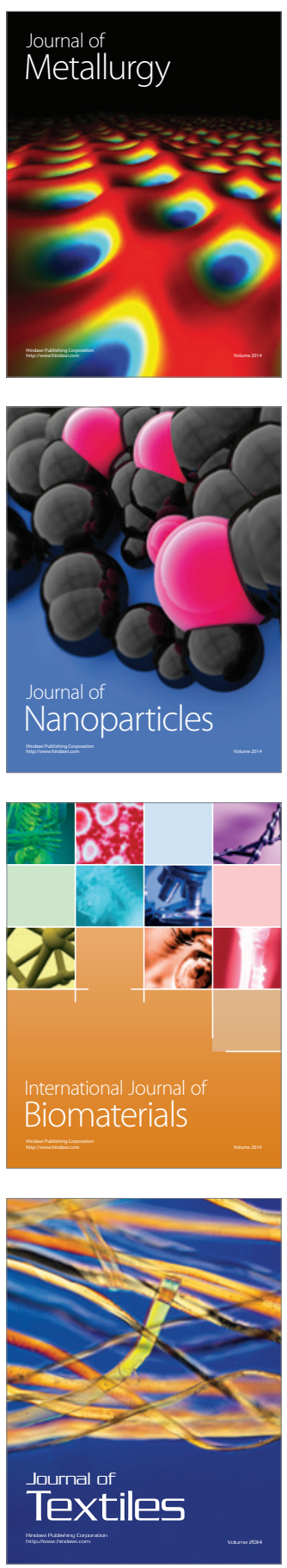